\title{
Modelo físico para a determinação de epicentro: uma ferramenta para 0 ensino da Sismologia
}

\author{
A. L. Melo', B. F. Chiba', E. N. S. Gomes ${ }^{1,2}$, C. B. SILLA', \\ 1 Faculdade de Geofísica- Instituto de Geociências/UfPa. Belém, Pará, Brazll \\ 2 Instituto Nacional de Clência e TeCnologia (INCT) da Criosfera

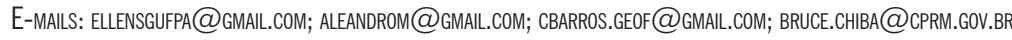

\begin{abstract}
This paper presents a small-scale physical model illustrating the determination of earthquake epicenters, which aims to contribute to the teaching of Geosciences in basic education. The model may be used as a didactic support in a ludic approach to the teaching of Seismology concepts in the classroom, as well as in laboratories, exhibitions, and museums. The experiment is built from accessible materials. The paper presents details about the construction of the model, the used materials, and the computer program used for data acquisition and processing. This model is part of the work done at the Analog Modeling Laboratory of the Geophysics Faculty at the Federal University of Pará, Brazil. The models developed there are presented yearly at the "What is Geophysics?" exhibition, which has aroused the interest of the general public. For the multidisciplinary character of its construction, the experiment may be used as didactic support for teaching concepts of Geology, Physics, Electronics, Mathematics, and Computing.
\end{abstract}

\author{
Manuscrito: \\ Recebido: 22/11/2017 \\ Corrigido: 28/03/2018 \\ Aceito: 24/04/2018
}

Citation:.Melo A.L., Chiba B.F., Gomes E. N. S., Silva C.B. 2018. Modelo físico para a determinação de epicentro: uma ferramenta para 0 ensino da Sismologia. Terræ Didatica, 14(2):173-184. URL: http://www.ige.unicamp.br/terraedidatica/.

Keywords: Epicenter location, Seismology, Geosciences teaching.

\section{Introdução}

Existe uma necessidade crescente da sociedade brasileira por profissionais na área de Geociências. Parte dessa demanda se deve a fatores como a grande província mineral do país e as descobertas de petróleo. No entanto, a procura pelas áreas de Geociências nas universidades é baixa. Acredita-se que isso se deva ao desconhecimento por parte da sociedade em geral sobre as Geociências e suas aplicações. Qualquer solução para atender a essa demanda passa pela divulgação e ensino das Geociências nas escolas. Entretanto, verifica-se que no currículo do ensino fundamental e médio as Geociências são pouco contempladas e diluídas nos currículos de Geografia e Ciências.

Um dos primeiros trabalhos a fazer uma discussão sobre a metodologia de ensino de ciências no primeiro grau mediante a experiência de Geologia com ênfase no campo foi Compiani (1989), neste trabalho foram investigados métodos e princípios relevantes para o ensino da Geologia do primeiro grau (atual ensino fundamental), além de uma discussão sobre a contribuição do fazer geologia para a aquisição do método científico no ensino. Uma análise crítica dos fatores que dificultam o ensino das Geociências na região Nordeste são apresentados em Santana \& Barbosa (1993). Estes vão desde a escassez de recursos didáticos na área voltados ao público do ensino médio até a precária formação dos professores. Esse panorama não é diferente em outras regiões do País.

No Brasil, nas últimas décadas há um movimento no sentido de melhorar a abordagem do ensino das Geociências no ensino básico. No que se refere a material didático na área, podemse citar projetos como o Geo-Escola (Carneiro et al. 2005, 2007), que disponibiliza material didático em CD-ROM ou website contendo informações geológicas, imagens e mapas com objetivo de servir de suporte computacional a professores do ensino fundamental e médio. O Geo-Escola vem sendo desenvolvido e sendo utilizado em escolas do ensino fundamental e médio ainda em caráter experimental. Outros projetos similares a GeoEscola vêm sendo desenvolvidos nos institutos de Geociências do país (Reis et al. 2005, Lopes \& Carneiro 2009 etc.) 
Existem, no país, iniciativas de capacitação de professores do ensino básico, no ensino das Geociências, a exemplo do que foi realizado no Departamento de Geociências Aplicadas ao Ensino (DGAE) do Instituto de Geociências da UNICAMP (Compiani et al. 2000, 2002). Apesar de extinto o Departamento, as atividades não se interromperam, graças à continuação do Programa de Pós-Graduação em Ensino e História de Ciências da Terra (PEHCT) do Instituto de Geociências da Unicamp, que foi completamente absorvido pela comunidade paulista de docentes de Geociências preocupados com questões educacionais.

No currículo do ensino básico brasileiro, os tópicos de Geociências desenvolvidos deixam a desejar. No fundamental (alunos entre 7 a 14 anos), alguns tópicos são abordados nas disciplinas de ciências e geografia. No ensino médio (alunos de 15 a 17 anos), tópicos das Geociências vêm sendo ministrados em disciplinas específicas (como Biologia, Química, Física e Geografia) e na parte interdisciplinar (Brasil 1999). Salvo raras exceções, os tópicos geocientíficos têm tido um tratamento fragmentado e disperso, sendo insuficiente para promover a compreensão da Terra como um sistema complexo e dinâmico para desenvolver a sensibilidade necessária para enfrentar os desafios impostos pela degradação ambiental, mostrando um caráter antropocêntrico e imediatista da filosofia de orientação dos conteúdos (Toledo 2005). Assim, há a necessidade de mais discussões em torno do tema do ensino de Geociências, além de trabalhos que contribuam com material didático.

Dentre as várias áreas de estudo das Geociências, a Sismologia, que tem por objeto o estudo dos sismos (terremotos), tem grande importância para a atividade humana, a exemplo de grandes catástrofes como: o tsunami na Indonésia (2004), os terremotos do Chile e Haiti (2011) e o terremoto do Japão (2011) que resultaram na perda de milhares de vidas e prejuízos incalculáveis para esses países com consequências em longo prazo. Em Sismologia, são estudadas as circunstâncias em que um abalo sísmico ocorre, suas causas e como eles estão distribuídos na Terra.

Este trabalho traz uma proposta de experimento que simula as condições, de forma simplificada, para a geração e detecção de abalos sísmicos. Este faz parte do conjunto de experimentos físicos criados e montados pelo Laboratório de Modelagem Analógica da Faculdade de Geofísica da Universidade Federal do Pará, que são apresentados na exposição itinerante "O que é Geofísica?”, criada em 2006 por professores e alunos da Geofísica da UFPA e tem por objetivo a divulgação das Geociências, em especial, a Geofísica e suas aplicações, com público-alvo o ensino básico, além da comunidade em geral.

O experimento apresentado neste Trabalho é constituído de duas partes, uma física e outra computacional, e pode ser facilmente construído em ambiente escolar. A parte física é constituída de uma fina placa de acrílico (que simula a crosta terrestre onde ocorre o evento sísmico) e dos sensores (que simulam "os sismógrafos", necessários para detectar os abalos sísmicos que se propagam na placa de acrílico). O registro e tratamento do sinal medido são feitos por meio de um computador utilizando um programa desenvolvido em Object Pascal no ambiente Delphi.

O programa de computador, seu executável e manual estão disponibilizados na Revista Terrce Didatica em Materiais Suplementares (MS). O programa não requer instalação e para utilizá-lo basta duplo clique. O computador precisa ter o sistema operacional do Windows instalado.

\section{Objetivos}

Ainda que o experimento proposto aqui, só tenha sido utilizado em exposições e feiras científicas, onde os visitantes eram convidados a interagir com o mesmo, por sua característica lúdica e a possibilidade de envolvimento do aluno desde a sua construção até sua utilização, ele tem potencial para ser utilizado como uma ferramenta didática no ensino da sismologia além de poder ser utilizado de forma multidisciplinar no ensino de disciplinas como: física, matemática, eletrônica, etc.

Sugerimos várias formas de utilização do experimento. Focando, inicialmente no ensino de sismologia, esse experimento pode ser utilizado no ensino de vários tópicos desenvolvidos no ensino fundamental e médio do Brasil, como por exemplo: no ensino fundamental inicial (alunos de 7 a 9 anos), na matéria Geografia, em temas como:

-Um olhar sobre o relevo terrestre, as placas Tectônicas e os impactos causados pelos Terremotos e Tsunamis, que tem por objetivos levar o aluno a: conceituar placas tectônicas, terremotos e tsunamis; analisar quais são as áreas de maiores instabilidades tectônicas; comparar os lugares de maior ocorrência de terremotos e tsunamis e seus principais impactos naturais, econômicos e sociais (tema da disciplina 
Geografia do ensino fundamental inicial, alunos de 7 a 9 anos);

I - Um olhar sobre o relevo terrestre, as placas Tectônicas e os impactos causados pelos Terremotos e Tsunamis, que tem por objetivos levar o aluno a: conceituar placas tectônicas, terremotos e tsunamis; analisar quais são as áreas de maiores instabilidades tectônicas; comparar os lugares de maior ocorrência de terremotos e tsunamis e seus principais impactos naturais, econômicos e sociais (tema da disciplina Geografia do ensino fundamental inicial, alunos de 7 a 9 anos);

II - Paisagem e Ambiente, que tem por objetivos levar o aluno a: compreender os terremotos como parte da dinâmica natural da Terra; estabelecer a relação entre os sismos e os danos que provocam. (tema da disciplina Geografia do ensino fundamental inicial, alunos de 7 a 9 anos);

III - A dinâmica da Terra, tópico da disciplina de geografia do ensino médio, que tem por objetivos: compreender o que são abalos sísmicos; verificar que a ocorrência de terremotos está diretamente relacionada ao movimento das placas tectônicas; reconhecer que o movimento das placas ocorre de forma convergente, divergente ou lateral (transformante); reconhecer que existe uma área da Terra mais propícia para ocorrência de terremotos; analisar as conseqüências dos terremotos; relacionar os terremotos com os tsunamis.

A curiosidade e interesse do aluno serão despertados por meio da brincadeira de determinar a região no experimento físico onde se originou uma perturbação, a partir apenas do registro dessa perturbação, a semelhança do mundo real. Mais informações sobre Geofísica, Sismologia e placas tectônicas pode ser obtidos em Teixeira et al. (2005).

A utilização do experimento proposto, bem como sua construção e utilização, podem ser feitas de acordo com os exemplos acima, mas o modelo pode também permitir o desenvolvimento de tópicos interdisciplinares, como por exemplo:

- Propagação de ondas (na disciplina de Física);

- Localização de objetos por triangulação (Matemática, Cartografia em Geografia);

- Conversão de pressão em eletricidade (Física);

- Tratamento do sinal digital (Física ou Matemática).

Assim, este trabalho tem por objetivo contribuir com uma proposta de ferramenta didática, lúdica e criativa para desenvolvimento de conceitos de Sismologia de maneira fácil e interativa. Além disso, devido às múltiplas atividades envolvidas na confecção do experimento, noções de física, matemática, eletrônica e programação podem ser exploradas. O experimento apresentado pode ser aperfeiçoado por professores e alunos.

\section{Materias e Métodos}

A crosta terrestre é a parte mais superficial da Terra sólida, constituída por rochas fraturadas e menos quentes que as do interior terrestre. A crosta recobre o manto, sendo dividida em diversas placas tectônicas que possuem densidades e espessuras variadas e estão em constante movimento. As interfaces de contato entre as placas, denominadas falhas tectônicas, são as regiões geológicas de origem dos terremotos devido aos grandes esforços ocasionados pelos confrontos e acúmulo de energia potencial. $\mathrm{O}$ ponto de origem do terremoto no interior da crosta terrestre denomina-se hipocentro e o ponto na superfície da Terra acima do hipocentro denomina-se epicentro. A observação e a análise dos terremotos possibilitam o estudo da Terra de forma global, desde a superfície até o núcleo, a partir das ondas sísmicas, originadas nos hipocentros, que viajam por todo o globo terrestre.

No experimento proposto, para torná-lo mais simples e didático, foram feitas algumas suposições estruturais da crosta terrestre, são elas: (1) geometria é plana; (2) sua espessura é delgada; (3) não há falhas geológicas; (4) a densidade de massa é constante.

Com as devidas simplificações, a crosta terrestre pode ser representada por uma placa contínua e delgada com densidade homogênea.

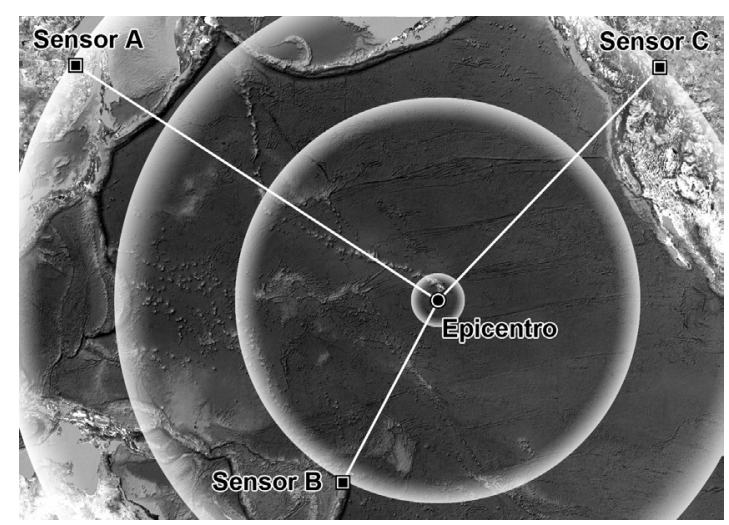

Figura 1. Esquema do experimento. É apresentado o esboço da propagação da onda a partir do epicentro. A perturbação se propaga até os sensores A, B e C 
Dessa forma, um impacto mecânico gerado na superfície da placa, simulando um evento sísmico, produz uma onda de superfície livre que se propaga com velocidade constante, radialmente a partir do ponto do impacto (o epicentro), simulando assim uma onda sísmica direta (Fig. 1). A espessura delgada garante que pouca reverberação ocorra no interior da placa e devido as suas dimensões físicas há ocorrência de ondas refletidas nas bordas, entretanto, apenas a onda direta é medida. A onda propagada pode ser observada em qualquer ponto na superfície da placa e sua observação é realizada por intermédio de sensores, que simulam os sismógrafos de componentes verticais (que medem apenas a componente vertical da perturbação), que estão localizados em pontos fixos e conhecidos, distribuídos sobre a superfície. Os sensores, por sua vez, permitem determinar os tempos de propagação da onda, possibilitando a estimação do epicentro. Para produzir o impacto mecânico, um simples bastão com ponta fina pode ser utilizado.

Para minimizar o uso de circuitos eletrônicos especiais para a aquisição e o processamento dos sinais observados, foram utilizados os recursos disponíveis em um micro computador de uso geral. Em todos os microcomputadores do tipo PC, são disponíveis entradas de áudio para o uso de microfones externos. Estas entradas podem ser usadas, com os devidos cuidados, em experimentos diversos que necessitem a medição de baixos níveis de voltagens. O sinal analógico disponível na entrada de áudio é convertido em sua versão digital com resolução de 8 ou 16 bit. A partir desse ponto o experimento é realizado por processamento digital com o uso de um programa adequadamente projetado, que será discutido mais a frente.

Para a detecção do "epicentro" (ponto de impacto) no modelo em escala reduzida, Figura 2, foi implementada da seguinte forma:

1. A maquete do experimento é constituída por uma placa de acrílico que simula a crosta da Terra e por três sensores fixos (a direita da Figura 2).

2. O evento sísmico é simulado usando um bastão com a ponta fina para a concentração da energia de impacto. Qualquer material pode ser usado, entretanto, a velocidade de propagação é função dos espectros do sinal de impacto gerado. Recomenda-se usar fontes com pouca duração. Nos testes experimentais, foram utilizadas canetas esferográficas como

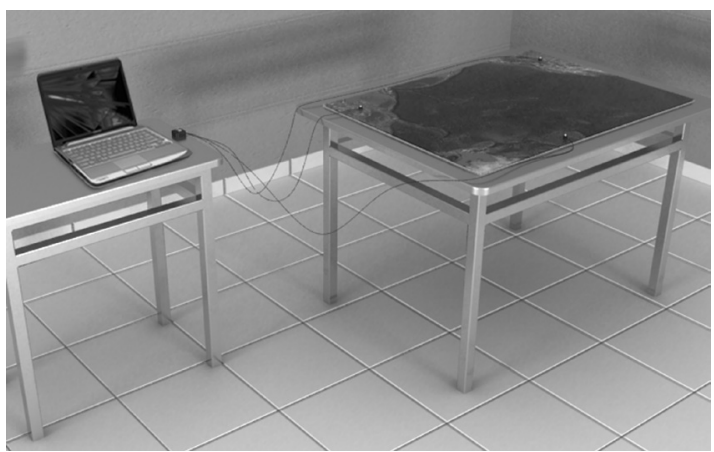

Figura 2. O conjunto maquete com sensores ligados a chave comutadora (imagem a direita) e está ligada ao computador (imagem a esquerda) que gerencia a aquisição e realiza os cálculos para a localização do epicentro.

fonte de impacto. Estas foram deixadas cair com a ponta sobre o acrílico.

3. Para a observação e detecção do epicentro são necessários três sensores fixados na placa (ver na Figura 2). Porém, devido às limitações de hardware do computador, apenas dois canais de áudio podem ser utilizados na aquisição dos dados simultaneamente, impedindo que a perturbação gerada seja registrada nos três sensores simultaneamente. Este problema pode ser solucionado realizando a aquisição em duas etapas e tomando um dos sensores como referência para ajustar os sinais registrados. Se as duas perturbações geradas são aproximadamente iguais em espectro os tempos de propagação são também aproximadamente iguais, produzindo pouco erro na estimação do epicentro.

$\mathrm{Na}$ Figura 3, tem-se o detalhe do sensor fixo na placa de acrílico.

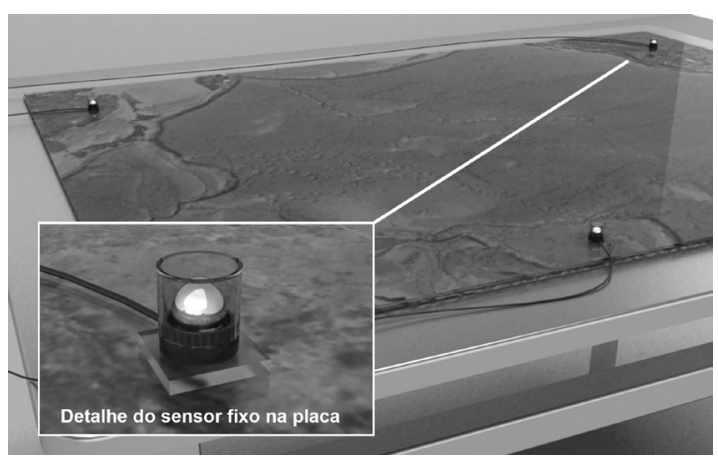

Figura 3. Maquete que simula a superfície da Terra e sobre a qual são produzidas as perturbações (sismos). Na placa de acrílico estão fixados os três sensores. Detalhe de um dos sensores utilizados na detecção das vibrações na maquete. Eles são fixados na placa de acrílico por uma fita dupla face de silicone. 


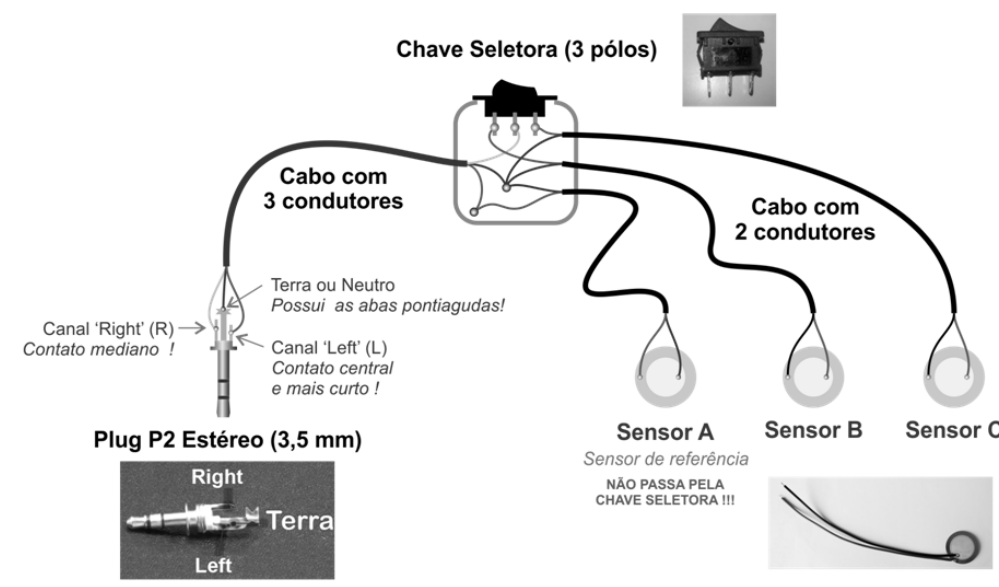

Figura 4. Desenho esquemático que mostra como os sensores estão ligados a chave comutadora e esta por sua vez está ligada aos cabos conectados do Conector do tipo P2 estéreo (com dois canais) no computador. interface gráfica (Fig. 5) por meio da qual são visualizados os sinais adquiridos (Fig. 5 item5), o mapa com a localização dos sensores sobre a placa de acrílico (Fig. 5 item1) e os controles necessários para o cálculo do epicentro (itens de 3 a 7 da Fig. 5).

Com esta interface propomos uma forma automatizada de calcular a região do epicentro. No Manual de instalação e funcionamento do programa, são dadas as informações sobre como instalar e utilizar o programa usado no experimento. Este Manual faz parte dos Dados Complementares do artigo que está disponível na Revista Terre

$\mathrm{Na}$ Figura 4, tem-se um desenho esquemático do funcionamento dos sensores. Um dos sensores é conectado diretamente a um dos canais da entrada de áudio (na Figura 4, o sensor A), enquanto que os outros dois sensores são conectados a uma chave comutadora (na Figura 4, os sensores B e C), de tal modo que apenas um sensor seja conectado ao outro canal da entrada de áudio por vez. Dessa forma, a entrada de áudio recebe dois sinais analógicos simultâneos e os converte na forma digital.

A aquisição e o processamento dos sinais são realizados no domínio digital, sendo necessário um programa de computador que controle a entrada de áudio, a leitura do sinal na forma digital e os cálculos matemáticos para a estimação da posição do epicentro. O programa Epicenter v.2 (disponível em Terre Didatica como Material Suplementar MT) foi desenvolvido no ambiente Delphi. Este programa gerencia, por meio da placa de som do computador, os sinais captados pelos sensores e detecta o instante em que o sinal (a onda) passa pela posição de cada sensor sobre a placa. A metodologia usada para a estimação do epicentro é baseada na triangulação geométrica apresentada mais adiante.

Para facilitar a interação com o usuário o programa Epicenter v.2 possui uma

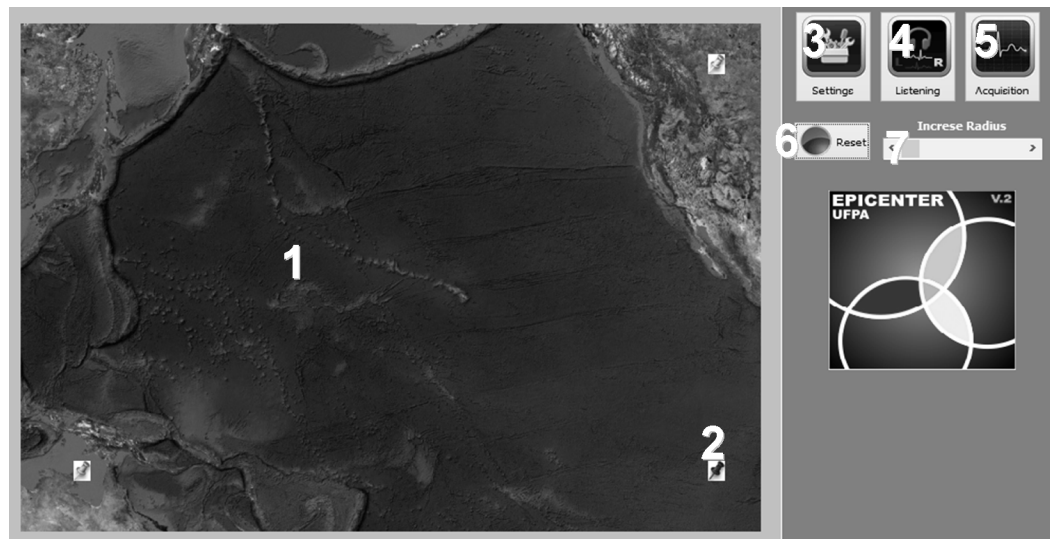

Figura 5. Interface gráfica do programa Epicenter v. 2 que capta o sinal e calcula a região do epicentro. Os componentes da interface estão enumerados de 1 a 7.

TERRAE DIDATICA 14-2, 2018

ISSN 1679-2300 


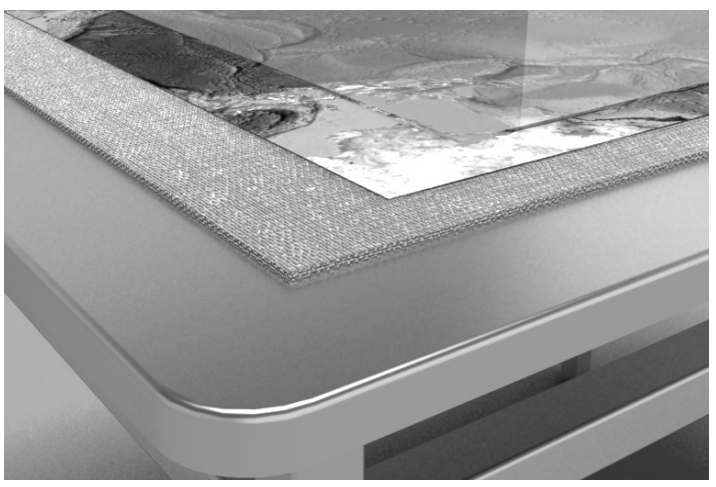

Figura 6. Componentes da maquete: placa de acrílico cristal, arte impressa em lona e espuma.

resistência mecânica necessária para suportar os impactos na geração da perturbação, a leveza para permitir o fácil manuseio e transporte, além de permitir o uso de arte impressa como ilustração por baixo da placa, dando assim mais realidade ao experimento. A placa repousa sobre uma fina camada de espuma macia com $5 \mathrm{~mm}$ de espessura, cuja função é isolá-la de possíveis vibrações que se propagam através da mesa de suporte da maquete, Figura 5. A espuma utilizada deve ser rígida o suficiente para suportar o peso da placa de acrílico e da arte, mas macia para dissipar as vibrações. A mesa de suporte da maquete deve ser plana, horizontal e estar em perfeito equilíbrio. Entre a placa de acrílico e a espuma é usada a arte gráfica impressa. Neste experimento estamos utilizando como arte uma ilustração da região conhecida como Círculo de Fogo do Pacífico, onde a ocorrência de abalos sísmicos é mais frequente devido ao encontro de várias placas tectônicas (Figs. 7 e 8). A ilustração está disponível no material suplementar do artigo que está disponível na Revista Terre Didatica.

Três sensores são utilizados para a localização do epicentro. A posição de cada sensor é conhecida e medida em relação a um dos cantos da placa de acrílico, que será a origem do sistema de coordenadas (Fig. 9). Estas informações são carregadas por meio da janela SETTINGS na interface gráfica (na Figura 5 item 3) do programa. A posição dos sensores pode ser carregada no programa de maneira visual ou manual (detalhes de como proceder para fazer a localização dos sensores é dada no Manual de instalação e funcionamento do programa no MS da Revista Terrae Didática. Os sensores devem estar colados na placa de acrílico e irão reagir ao movimento local provocado pela passagem do sinal gerado pela fonte. Para

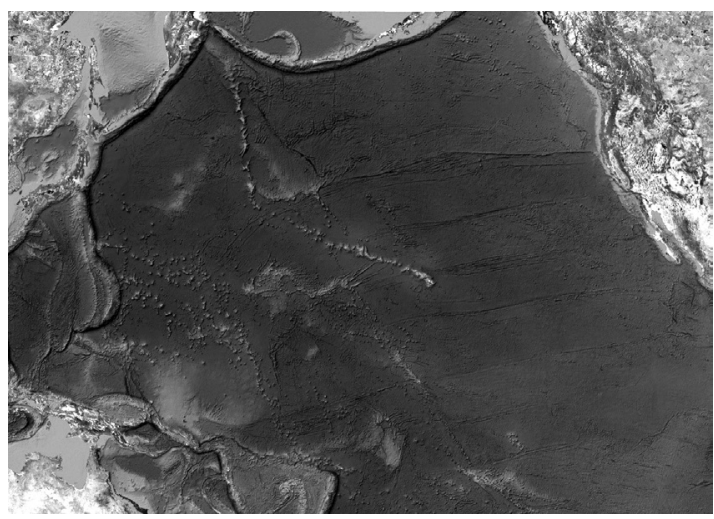

Figura 7. Ilustração que mostra as placas tectônicas. Na área destacada tem-se a Região conhecida como Círculo de Fogo do Pacífico, mostrada em detalhes na Figura 8. Retirada de https://eu.wikipedia.org/ wiki/Pazifikoko_plaka.

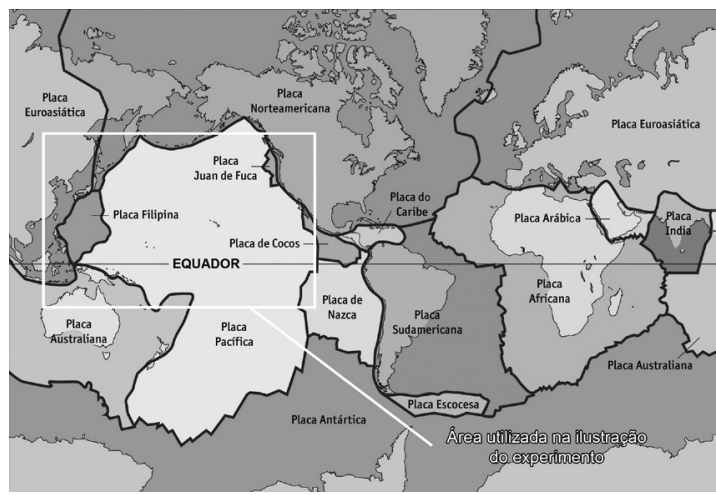

Figura 8. Região conhecida como Círculo de Fogo do Pacífico, onde a ocorrência de abalos sísmicos é mais frequente devido ao encontro de várias placas tectônicas. Retirada do Google Maps

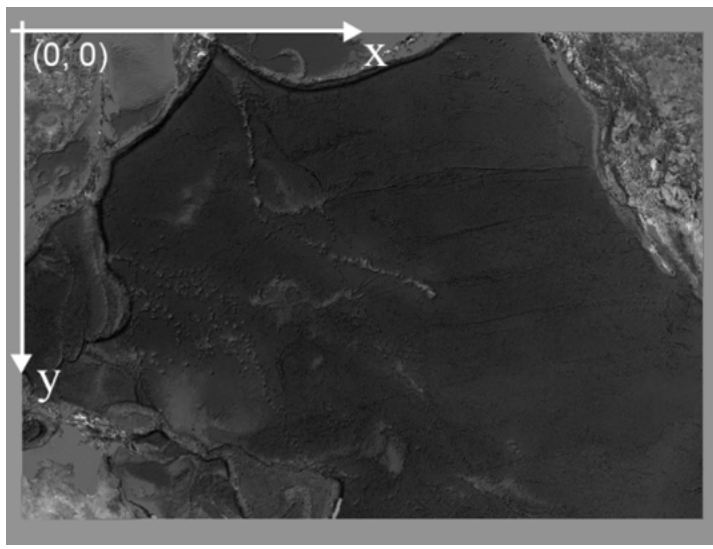

Figura 9. O canto superior esquerdo é tomado como origem das coordenadas na placa. A posição da cada um dos sensores deve ser tomada em relação a essa origem e essa posição deve ser carregada na interface gráfica, Figura 5 item 3 (na janela Settings, para mais detalhes ver Manual de instalação e funcionamento do programa. 
proporcionar o máximo acoplamento mecânico os sensores são colados na placa com fita dupla face de silicone (Fig. 3). Nenhum outro objeto, além dos sensores, deve ser colocado sobre o acrílico, para evitar deformações e variação na velocidade da onda. Cada sensor é construído utilizando um disco piezoelétrico (buzina) que converte uma vibração mecânica (deformação) em sinal elétrico que é lido na forma de diferença de potencial em uma escala de centenas de milivolts. Para baixar a frequência de ressonância natural do disco piezoelétrico, uma esfera de aço com massa de 17 g e diâmetro de $16 \mathrm{~mm}$ é centrada sobre o disco pizoelétrico (fixada com cola de silicone). Um encapsulamento de acrílico é encaixado na base plástica envolvendo o conjunto disco e esfera (Fig. 11), isolando-os das interferências externas, tais como som ambiente e contato externo Os três sensores são conectados a uma ou chave seletora de 3 polos (Fig. 12) permitindo a leitura simultânea de dois sensores, sendo que um dos sensores (usamos o sensor A) é utilizado duas vezes e serve como sensor de referência no cálculo das diferenças de tempo de chegada do pulso. O cabo elétrico usado nas conexões deve ser do tipo blindado e de baixa impedância, semelhante aos utilizados em fones de ouvido de boa qualidade (ver no MS Sugestão de material a ser utilizados no experimento). A chave seletora, por sua vez, é conectada na entrada de microfone do PC usando um conector P2 estéreo (Fig. 13). O esquema de ligação entre os sensores, chave comutadora e o conector P2 estéreo é indicado na Figura 4.

O sinal é registrado em um computador (desktop ou notebook) equipado com placa de som com microfone estéreo (entrada para dois canais: esquerdo e direito). O microfone estéreo é fundamental para na aquisição, pois permite a leitura simultânea em dois canais, com um dos sensores servindo como referência. A entrada de microfone na placa de som genérica pode receber sinais elétricos com amplitudes que variam de -1 a 1 volt. Algumas placas de som são gerenciadas por aplicativos que ajustam os níveis de sensibilidade da placa, simulam efeitos de ambiente, equalizam o sinal de saída, etc, tais funcionalidades extras devem estar desabilitadas para proporcionar uma aquisição de sinal mais confiável. O computador utilizado no experimento tem a seguinte configuração: Sistema operacional Windows XP ou posterior; $4 \mathrm{Mb}$ de espaço em disco para armazenamento dos arquivos do programa; $512 \mathrm{Mb}$ memória RAM; Vídeo em

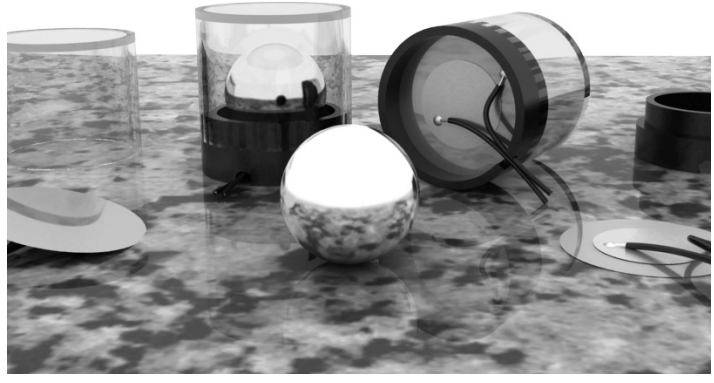

Figura 10. Detalhes das componentes do sensor.

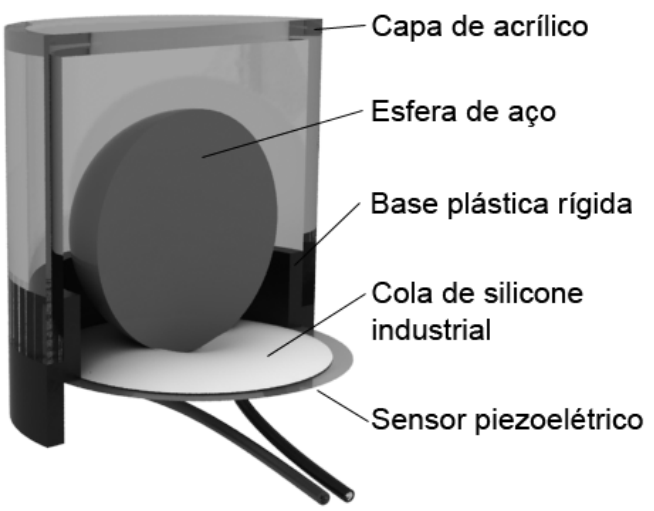

Figura 11. Descrição das componentes do sensor

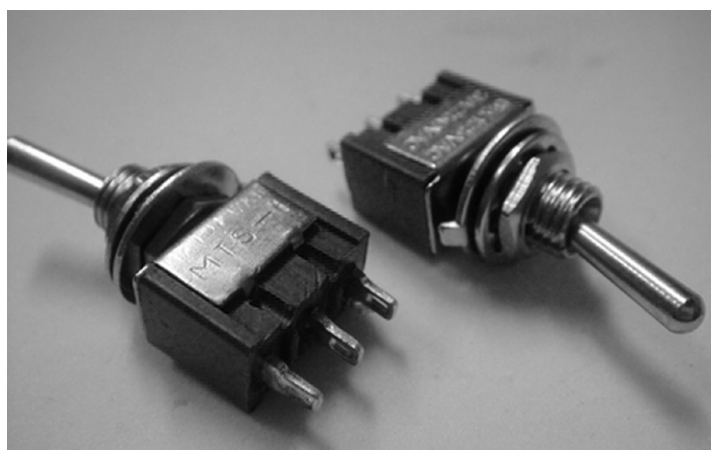

Figura 12. Chave comutadora de duas posições e três contatos.

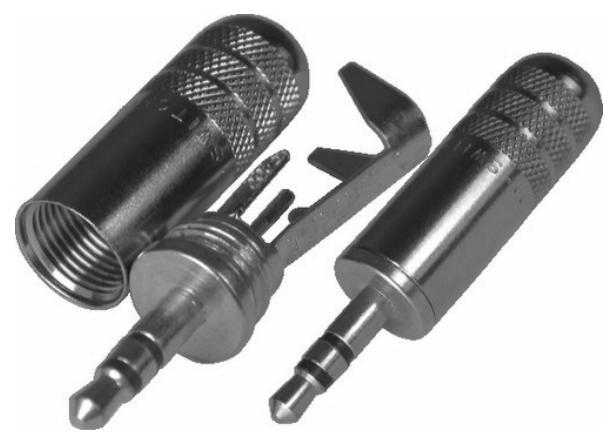

Figura 13. Plug P2 estéreo 


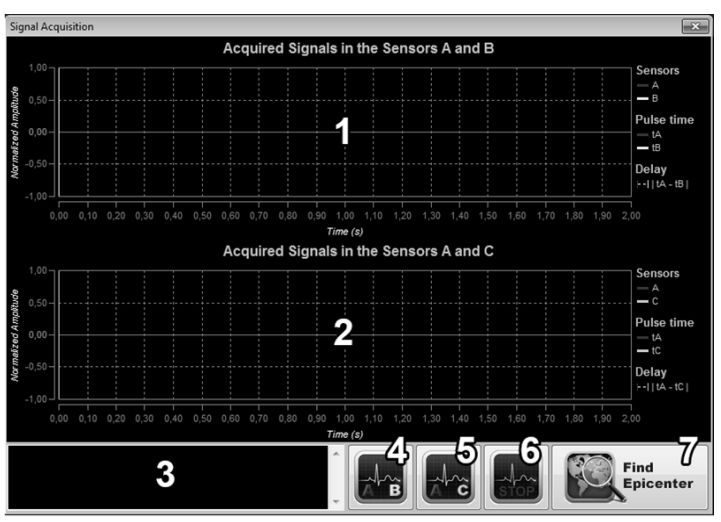

Figura 14. Janela do Signal Acquisition que aparecerá na tela do computador após o botão de Acquisition for acionado. Nesta janela são mostrados o sinal adquirido no par de sensores $A B$ (item 1) ou no par AC (item 2). No Item 3 é mostrado o status na aquisição. Nos itens 4 e 5 escolhe-se que par de sensores medirá o sismo. 0 botão do item 6 só é utilizado no caso de aquisição com picking manual (para mais informações consultar Manual de instalação e funcionamento do programa). No item 7 o epicentro determinado.

resolução 800x600 dpi; Mouse e Placa de som com entrada para microfone estéreo. O programa Epicenter v.2 roda em sistema operacional Windows ${ }^{\circledR}$ e possui três arquivos: o executável Epicenter.exe, arquivo de configurações config. ini e a imagem utilizada como ilustração na maquete default.jpg.

\section{Experimento}

A aquisição do evento ("terremoto") deve ser realizada em duas etapas. A pessoa que está realizando o experimento deverá clicar no botão Acquisition (item 5 da Fig. 5). A janela Signal Acquisition (Fig. 14) aparecerá na tela. Clicando o botão $\mathrm{AB}$ ativa (pressionando o botão "AB", item 4 da Fig. 14) o primeiro par de sensores (sensores A e B), que ficará em modo de escuta por cinco segundos. Durante este intervalo deve-se, com o auxílio de um bastão-fonte (caneta esferográfica), gerar a perturbação em um ponto da placa de acrílico (Fig.15). A aquisição é considerada bem sucedida quando, o programa detecta no sinal registrado o instante de chegada do pulso (Fig. 16).

Na Fig. 16, o pulso é detectado a partir da amostra cuja amplitude é maior ou igual a um valor estabelecido nas conFig.ções de ajuste do programa Epicenter.exe. $O$ valor de referência pode ser calculado automaticamente pelo programa e

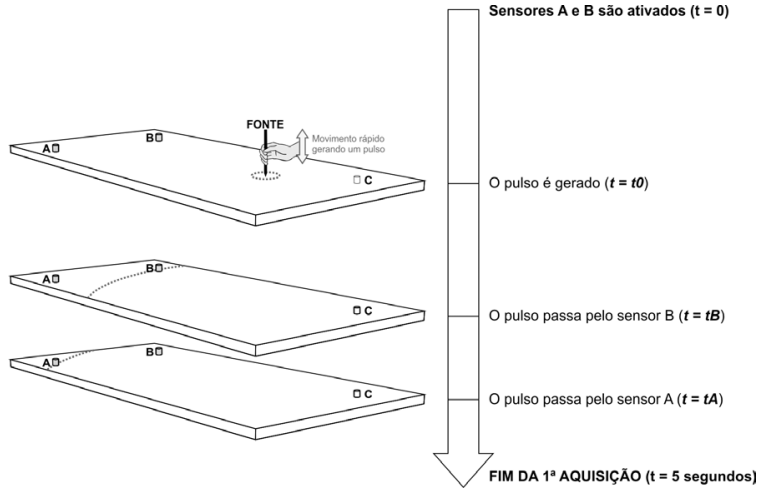

Figura 15. Desenho do esquema que mostra a $1^{\text {a }}$ aquisição.

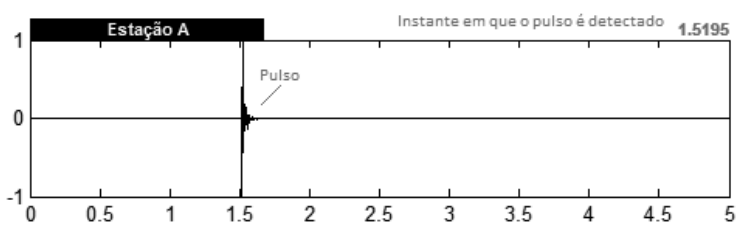

Figura 16. Exemplo de sinal adquirido no sensor A com o instante da passagem do pulso detectado.

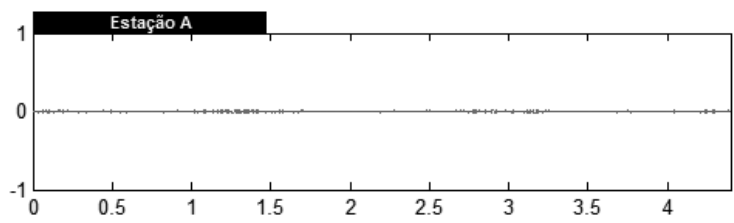

Figura 17. Exemplo de sinal ruidoso adquirido em ambiente com algumas pessoas conversando. Notar que a amplitude do ruído é bem menor que a amplitude da perturbação gerada sobre a placa (comparar com a Figura 16).

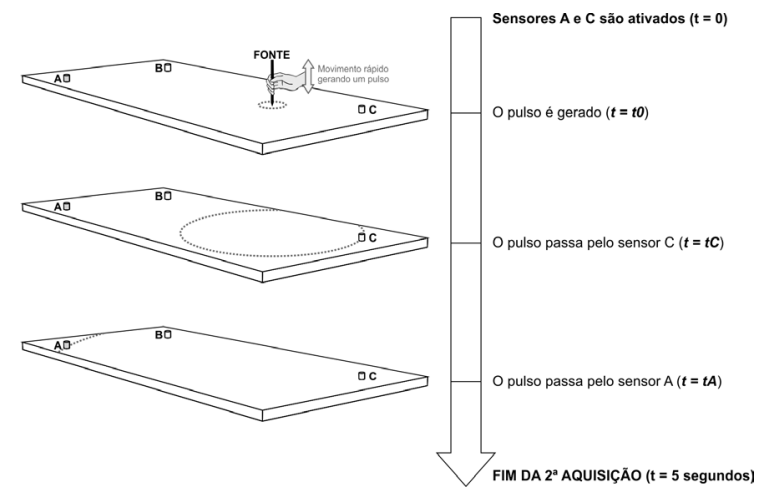

Figura 18. Esquema que mostra a $2^{\mathrm{a}}$ aquisição.

depende das condições ambientes. O procedimento para o cálculo do valor de referência é apresentado no Manual de instalação e funcionamento do programa disponibilizado como MS na Revista.

São considerados ambientes ruidosos aqueles lugares com grande fluxo de pessoas e/ou próximos 
a fontes sonoras de grande amplitude (na Fig. 17 exemplo de registro de ruído).

Se a aquisição no primeiro par de sensores for bem sucedida, deve-se acionar (pressionando o botão "AC", item 5 da Fig. 14) o segundo par de sensores (sensores A e C) e gerar mais uma perturbação o mais próximo possível do ponto escolhido para a primeira perturbação, sempre com o auxílio da mesmo bastão (fonte) e aproximadamente na mesma intensidade (Fig. 18).

Durante a aquisição do sinal é importante ficar atento à chave comutadora para que haja correspondência entre os pares de sensores ativos na maquete e os pares de sensores ativos no programa Epicenter.exe, dependendo do par de sensores ativos, $\mathrm{AB}$ ou $\mathrm{AC}$, o item 1 ou 2 estará respectivamente ativado na Fig. 14. Por fim presione o botão Find Epicenter para encontrar o epicentro. O procedimento de determinação do epicentro descrito acima é conhecido no Manual como aquisição com picking automático. Há também no programa da opção da determinação do epcentro com picking manual, que depende da interferência do usuário que esta realizando a aquição. Para detalhes ver o Manual de instalação e funcionamento do programa.

Após a aquisição dos sinais, o programa calcula os raios dos círculos centrados nas posições das estações. O expositor deverá fazer o ajuste (incrementar) dos raios até a interseção dos três círculos. Esse ajuste é feito por meio de uma barra de rolagem na interface gráfica do programa (item 7 da Fig. 5) da seguinte forma: o expositor arrasta a barra de rolagem até que os três círculos formem uma área de interseção que conterá o ponto onde a perturbação foi gerada (na Fig. 19).

\section{Método de determinação da região do epicentro}

Partindo do pressuposto de que a placa de acrílico se comporta como um meio uniforme (isotrópico e homogêneo), e qualquer perturbação na superfície da placa de acrílico irá se propagar em todas as direções com a mesma velocidade, as distâncias $d_{A}, d_{B}$ e $d_{C}$ (Fig. 20) percorridas pelo pulso (onda) a partir do epicentro até cada um dos sensores A, B e C são dadas por:

$$
\begin{aligned}
& d_{A}=V\left(t_{A}-t_{0}\right), \\
& d_{B}=V\left(t_{B}-t_{0}\right), \\
& d_{C}=V\left(t_{C}-t_{0}\right),
\end{aligned}
$$

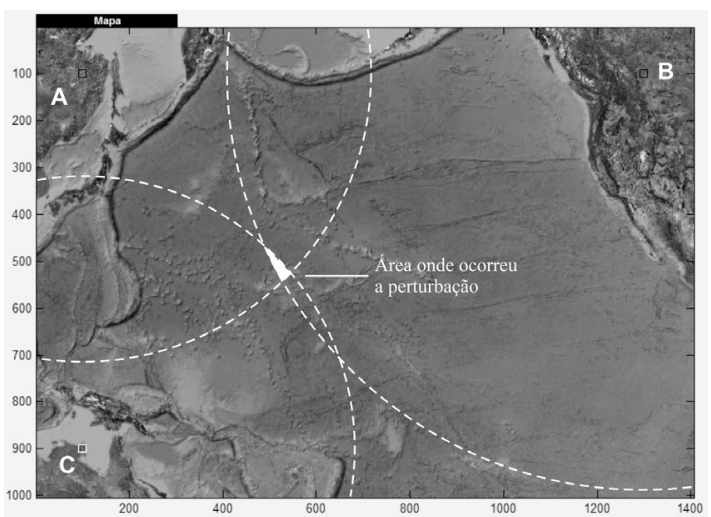

Figura 19. A área de interseção entre os círculos determina a área do epicentro

em que: $t_{0}$ é o instante em que o pulso foi gerado, $t_{A}, t_{B}$ e $t_{C}$, são instantes de chegada do pulso em cada sensor e $V$ é a velocidade do pulso. Como o instante $t_{0}$ em que o pulso foi gerado é desconhecido, ele não pode ser medido no experimento e as distâncias $d_{A}, d_{B}$ e $d_{C}$ não podem ser calculadas. $\mathrm{O}$ que podemos fazer é, a partir dos instantes de chegada do pulso em cada sensor, calcular os intervalos de tempo tomando o sensor A como referência:

$$
\begin{aligned}
\Delta t_{A A} & =t_{A}-t_{A}=0, \\
\Delta t_{A B} & =t_{B}-t_{A}, \\
\Delta t_{A C} & =t_{C}-t_{A} .
\end{aligned}
$$

O intervalo relativo $\Delta t_{A A}$ na equação (2) será sempre nulo. Dependendo da posição onde o pulso foi gerado, os intervalos podem ser positivos ou negativos. Valores negativos ocorrem quando o sensor A está mais distante do epicentro que os outros sensores. Esses valores não podem ser usados no cálculo dos raios dos círculos para a triangulação do epicentro, pois não existem raios negativos. Esses valores negativos são evitados subtraindo-se de todos os intervalos relativos o menor valor entre eles, obtendo-se, assim, valores não negativos para os intervalos de tempo.

Se, por exemplo, os instantes de chegada do pulso nos sensores guardam a seguinte relação:

$$
t_{B}>t_{A}>t_{C} \cdot(3)
$$

Os intervalos de tempo relativos seriam então:

$$
\begin{aligned}
& \Delta t_{A A}=t_{A}-t_{A}=0, \\
& \Delta t_{A B}=t_{B}-t_{A}>0,(4) \\
& \Delta t_{A C}=t_{C}-t_{A}<0 .
\end{aligned}
$$


Subtraindo-se deles o menor valor, $\Delta t_{A C}$ , teremos:

$$
\begin{aligned}
& \Delta t_{A}=t_{A}-t_{A}-\left(t_{C}-t_{A}\right)=t_{A}-t_{C} \\
& \Delta t_{B}=t_{B}-t_{A}-\left(t_{C}-t_{A}\right)=t_{B}-t_{C},(5) \\
& \Delta t_{C}=t_{C}-t_{A}-\left(t_{C}-t_{A}\right)=0 .
\end{aligned}
$$

Em que: $\Delta t_{A}, \Delta t_{B}$ e $\Delta t_{C}$, são intervalos de tempo não negativos de acordo com a equação (3) e, ainda de acordo com esta relação, os raios dos círculos (Fig. 21) podem ser calculados da seguinte forma:

$$
\begin{aligned}
& r_{A}=V \Delta t_{A}=V\left(t_{A}-t_{C}\right), \\
& r_{B}=V \Delta t_{B}=V\left(t_{B}-t_{C}\right), \\
& r_{C}=V \Delta t_{C}=V\left(t_{C}-t_{C}\right)=0 .
\end{aligned}
$$

Tais valores de raio não são iguais às distâncias percorridas pelo pulso até os sensores na propagação direta, porém a diferença entre a distância de cada sensor e o epicentro, equação (1) e os respectivos raios, equação (6) é a mesma para todos eles, pois:

$$
\begin{aligned}
& d_{A}-r_{A}=V\left(t_{A}-t_{0}\right)-V\left(t_{A}-t_{C}\right)=V\left(t_{C}-t_{0}\right), \\
& d_{B}-r_{B}=V\left(t_{B}-t_{0}\right)-V\left(t_{B}-t_{C}\right)=V\left(t_{C}-t_{0}\right),(7) \\
& d_{C}-r_{C}=V\left(t_{C}-t_{0}\right)-0=V\left(t_{C}-t_{0}\right) .
\end{aligned}
$$

O epicentro está equidistante dos círculos centrados nos sensores e de raios dados na equação (6) da distância $r=V\left(t_{c}-t_{0}\right)$ . Independentemente das relações entre os instantes de chegada do pulso (ver equação (3)) os raios dos círculos em A, B e C podem ser reescritos por:

$$
\begin{aligned}
& r_{A}=V\left(t_{A}-t_{\text {min }}\right), \\
& r_{B}=V\left(t_{B}-t_{\text {min }}\right), \\
& r_{C}=V\left(t_{C}-t_{\text {min }}\right) .
\end{aligned}
$$

em que $t_{\min }$ é o menor valor entre $t_{A}, t_{B}$ e $t_{C}$.

A determinação do epicentro é realizada por

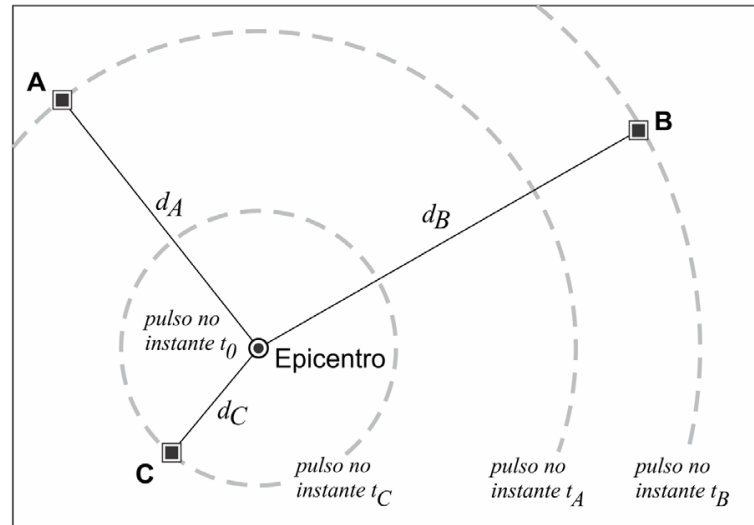

Figura 20. Distâncias $d_{A}, d_{B}$ e $d_{C}$ do epicentro para os sensors $A, B, e$, respectivamente. Tem-se as frentes de onda (círculos centrados no epicentro) chegando em cada sensor nos tempos $t_{A}, t_{B}$ e $t_{C}$.

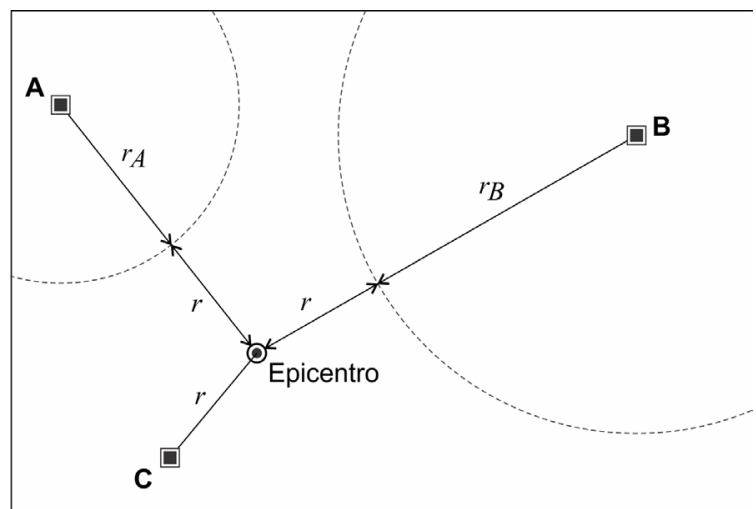

Figura 21. Raios $r_{A}, r_{B}$ e $r_{C}=0$, dos círculos centrados nos sensores $A, B$, e $C$, respectivamente. A distância do epicentro para cada círculo é de $\Delta r$.

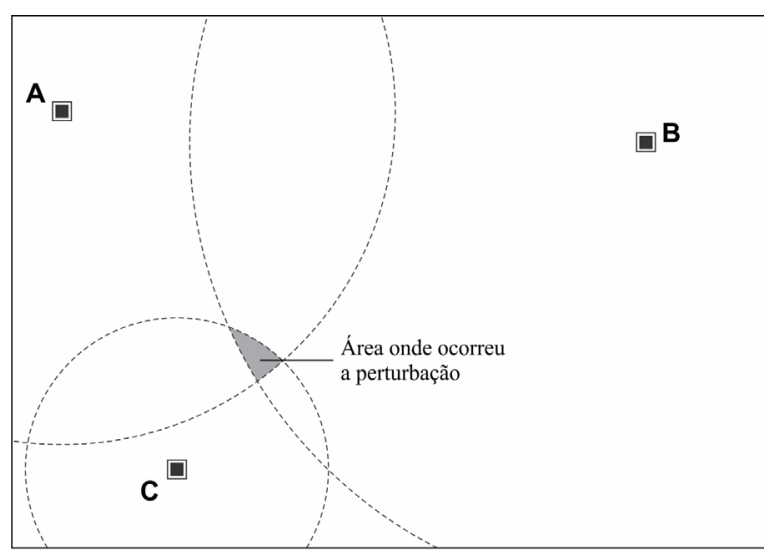

Figura 22. Após a expansão dos círculos centrados em $A$, $B$, e $C$, sua area de interceção determina a origem do "terremoto" 
meio do acréscimo de $\Delta r$ nos raios dos círculos centrados em A, B e C. Como o valor de $\Delta r$ é desconhecido, no experimento os raios dos círculos são aumentados de um incremento igual por meio da barra de rolagem da interface do programa, Figura 5- item 9, até a interseção destes. A área de interseção determina a região onde ocorreu a perturbação (Fig. 22, região do epicentro).

\section{Conclusões}

Este trabalho propõe uma ferramenta para o ensino da Sismologia, área que faz parte das Geociências, com objetivo de contribuir para quebra do paradigma da aprendizagem de forma passiva uma vez que envolve o aluno no processo de sua própria aprendizagem por meio de tarefas instigantes que estimulam a criatividade $\mathrm{e}$ inteligência. É apresentado um modelo em escala reduzida que determina a posição de epicentros que foi desenvolvido no Laboratório de Modelagem analógica da Faculdade de Geofísica da UFPa. Todas as etapas da construção e funcionamento do modelo são discutidas e desta forma, o mesmo pode ser reproduzido em qualquer ambiente escolar.

$\mathrm{O}$ experimento desperta o interesse para questões voltadas aos terremotos e suas causas, tendo sido um dos experimentos de maior procura na Exposição O queé Geofísica?, apresentada a alunos e professores do ensino fundamental.

O processo de construção envolve noções de física, matemática, eletrônica e programação; assim, o experimento além de ser utilizado para introduzir noções de sismologia, pode ter aplicações multidiciplinares na sala de aula.

A determinação da região do epicentro é feita pelo processo de triangulação dos sinais recebidos com um programa computacional desenvolvido em ambiente Delphi. A versão executável do programa, as instruções de instalação, a descrição de possíveis materiais a ser utilizados no experimento e o manual de utilização constituem material suplementar disponibilizado por Terre Didatica.

\section{Agradecimentos}

Os autores gostariam de agradecer ao Programa de Pós Graduação e Faculdade de Geofísica da qual fazem parte os professores e alunos envolvidos neste trabalho; ao PET-Geofísica e ao MEC pelas bolsas dos alunos do PET; ao Laboratório de
Modelagem Analógica da Geofísica - UFPA onde o trabalho foi desenvolvido, e à Sociedade Brasileira de Geofísica - SBGf, pelo apoio financeiro na montagem dos experimentos.

\section{Referências}

Carneiro C.D.R., Toledo M.C.M.de, Almeida F.F.M.de. 2004. Dez motivos para a inclusão de temas de Geologia na Educação Básica. Rev. Bras. Geoc. 34(4):553-560. URL: http://www.ppegeo.igc.usp. br/index.php/rbg/article/viewFile/9787/9135.

Carneiro C.D.R., Piranha J.M., Barbosa R. 2004b. Projeto Geo-Escola. Materiais Didáticos em Geociências com suporte no computador. Módulo São José do Rio Preto, State of São Paulo. São José do Rio Preto, UNESP and Unicamp (CD-ROM: Windows XP).

Carneiro C.D.R., Barbosa R. 2005. Geo-Escola: disseminação de conteúdos de Geociências por meio do computador para docentes de Ciências e Geografia no Nível Fundamental em Jundiaí-Atibaia, SP. Geologia USP - Série Didática, Publ. Espec., 3:7182, setembro 2005.

Carneiro C.D.R., Barbosa R., Piranha J.M. 2007. Bases teóricas do projeto Geo-Escola: uso de computador para ensino de Geociências. Rev. Bras. Geoc., 37(1):90-100. URL: http:/www.rbg.sbgeo.org.br/ index.php/rbg/article/view/A-1633/954.

Compiani M. 1989. O Fazer Geologia com Ênfase no Campo na Formação de Professores de Ciências para o $1^{\circ}$ grau ( $5^{a}$ a $8^{a}$ séries). Campinas: Unicamp. $238 \mathrm{p}$. (Masters Dissert.)

Compiani M., Cunha C.A.L.S. 1992. O ensino de Geociências nos 3 graus de escolaridade. Um panorama do Brasil. In: Congresso Geologia de España, 3, y Congresso Latinoamericano de Geologia, 8, Salamanca. Actas..., tomo I, p.324-352.

Compiani M., Figueirôa S. F. M., Gonçalves P. W., Newerla V.B., Nogueira A., Altomani A., Finco G., Silva F. K. M. 2000. Geociências e a Formação Continuada de Professores em Exercício no Ensino Fundamental: reflexões. Campinas, ProPosições, 1(4):25-35.

Compiani M., Figueirôa S. F. M., Gonçalves P. W., Nogueira A., Newerla V. B., Altomani A., Finco G., Silva F. K. M. 2002 . Projeto Geociências e a formação continuada de professores em exercício no ensino fundamental: reflexões e resultados finais. Zona Próxima, Colômbia, n. 3, p. 26-49.

Compiani M. 2006. Linguagem e percepção visual no ensino de Geociências. Pro-Posições, 17(1):85-104.

Lopes M.M. 1990. Contribution of Brazilian science museums to geological education. J. Geol. Educ., 38:460-462.

Lopes O.R., Carneiro C.D.R. 2009. O jogo "Ciclo das 
Rochas" para ensino de Geociências. Rev. Bras. Geoc., 39(1):30-41. URL: http://ppegeo.igc.usp. br/index.php/rbg/article/view/7627/7054. Acesso 12.12.2017.

Reis M.A.F., Carvalho C.V.A, Carvalho J.V., Rodrigues M.A.C., Medeiros M.A.M., Villena H.H., Oliveira F.M., Dornelas V.R. 2005. Sistema Multimídia Educacional para o Ensino de Geociências: uma Estratégia Atual para a Divulgação da Paleontologia no Ensino Fundamental e Médio. An. Inst. Geoc. UFRJ, 28(1):70-79.

Santana J C B, Barbosa L.M. 1993. A Realidade do En- sino de Geociências no $2^{\circ}$ Grau em Feira de Santana, Bahia. Rev. Bras. Geoc., 23(1):98-106.

Silva A., Vasconcelos A.C.B.C., Paschoale C. 1982. Uma base para elaboração do currículo de Geologia. [A basis for Geology curriculum development] In: Amaral I.A. org. 1982. Simpósio Nacional: O Ensino de Geologia no Brasil. Documento Final. Belo Horizonte: SBG, p. 71-91.

Teixeira W., Fairchild R. T., Toledo M.C.M.de, Taioli F. 2009. Decifrando a Terra. São Paulo. Cia. Edit. Nacional. 